\title{
Amino Imidate Catalyzed Asymmetric Michael Reactions of Ketones and Nitroalkenes
}

\author{
Bohdan Sosunovych \\ Alexander J. Brown \\ Paul A. Clarke* (i)
}

Department of Chemistry, University of York, Heslington, York, YO10 5DD, North Yorkshire, UK

paul.clarke@york.ac.uk

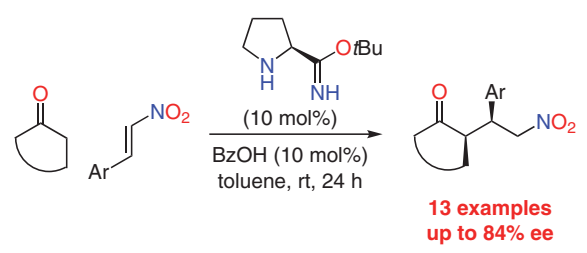

catalyst, generating products with enantioselectivities of $<25 \% .^{12}$ Highly functionalized and sterically bulky proline derivatives give products in much greater enantioselectivities of $60-95 \%$ but can involve protracted synthesis. ${ }^{13} \mathrm{We}$ recently reported the serendipitous discovery of a new type of pyrrolidine-containing organocatalyst, the tert-butyl imidate of L-proline (3), together with its ability to catalyze asymmetric aldol reactions in good to high enantiomeric excesses $^{14}$ (Scheme 1). In this paper, we further explore the scope of tert-butyl L-proline imidate (3) as an enantioselective organocatalyst for the Michael addition of cyclic ketones, heteroatom-containing cyclic ketones, or aldehydes to several nitroalkenes (Scheme 1).

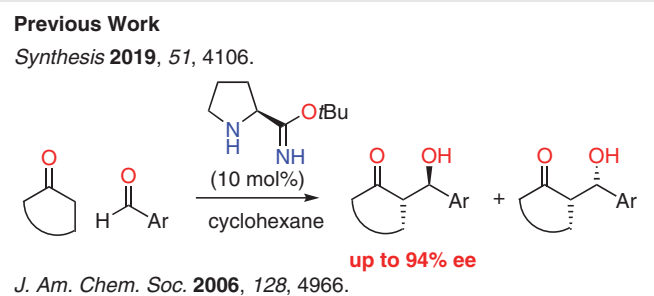
the award of the 2021 Nobel Prize in Chemistry, there has been considerable interest in the study of small organic molecules capable of catalyzing a wide range of synthetic transformations enantioselectively. The pyrrolidine ring is a privileged structure regarding catalytic ability, and it is often substituted with carboxylic acids, ${ }^{2}$ amides, ${ }^{4}$ esters, ${ }^{5}$ tetrazoles, ${ }^{6}$ or silyl ethers of tertiary alcohols. ${ }^{7}$ Each of these modifications bestows subtle changes in catalytic ability, enantioselectivity, diastereoselectivity, and the types of reaction which can be catalyzed. The most important of these reactions are aldol condensations, ${ }^{8}$ Diels-Alder cycloadditions, ${ }^{9}$ and Michael additions, ${ }^{8 a, b, 10}$ all of which lead to valuable chiral products from simple achiral starting materials. Michael reactions, especially those of nitroalkenes, have been of interest to several research groups. ${ }^{11}$ Proline itself was shown to be a relatively poor 
Our initial studies focused on the Michael reaction of cyclohexanone with $\beta$-nitrostyrene, as the reaction is well benchmarked in the literature and the products are well characterized. ${ }^{11 \mathrm{c}}$ In our earlier work on the amino imidatecatalyzed aldol reaction, it was noted that the reaction solvent played a significant role in both the conversion to products and the enantioselectivity of the reaction. Therefore, a screen of reaction solvents was undertaken with this standard reaction.

Cyclohexanone (1a) and $\beta$-nitrostyrene (2a) were stirred in the presence of $10 \mathrm{~mol} \%$ of tert-butyl L-proline imidate (3) at room temperature in a variety of solvents (Table 1). As can be seen, the reaction proceeded poorly when highly dipolar aprotic solvents (Table 1 , entries 1 and 2 ) or polar protic solvents (entries 11-13) were used. The other solvents, both dipolar and nonpolar, all gave reasonable to excellent conversions, generally above $90 \%$ (entries $4-10$ ). The reaction was the most diastereoselective when a nonpolar solvent such as cyclohexane, $\mathrm{CH}_{2} \mathrm{Cl}_{2}$, or toluene was used (entries 6, 8, and 10), giving syn/anti ratios of greater than 9:1. Determination of the enantioselectivity of the reactions showed that nonpolar hydrocarbon solvents were preferable, generating products with the highest enantioselectivities of $39 \%$ and $43 \%$ for cyclohexane and toluene respectively (entries 6 and 10). The absolute stereochemistry $(1 R, 2 S)$ of the major (syn) diastereomer was confirmed by comparison of its optical rotation and HPLC retention times with literature data. ${ }^{11,, 15}$ The other solvents led to products with much lower enantioselectivities. These trends can be rationalized by the general preference for nonpolar solvents in the synthesis of enamines from ketones and amines, and by the ability of highly dipolar or polar protic solvents to form competing hydrogen bonds with the amino imidate catalyst, which can disrupt the formation of hydrogen bonds between the catalyst and the substrate, essential for enhanced enantioselectivity. Although the catalyst was not recovered from these reactions, it was clearly visible in the ${ }^{1} \mathrm{H}$ NMR spectrum of the crude reaction mixture and there was no evidence of its hydrolysis to proline amide. These results, combined with solubility considerations of the substrates, led to the selection of toluene as the solvent of choice.

In an attempt to increase the enantioselectivity of the reaction, two changes were made: (i) the temperature was reduced to $0{ }^{\circ} \mathrm{C}$, and (ii) $20 \mathrm{~mol} \%$ of catalyst was used. In the reaction where the temperature was lowered to $0{ }^{\circ} \mathrm{C}$, the conversion dropped to $60 \%$ and the enantioselectivity of product 4 a dropped to $27 \%$, while the syn/anti ratio increased to $10.3: 1$. Increasing the catalyst loading to $20 \mathrm{~mol} \%$ had no effect on either the diastereoselectivity or enantioselectivity of the reaction.

With these results in hand, we examined the scope of the ketone partner (Scheme 2). Tetrahydropyran-4-one (1b) underwent a smooth and complete reaction in 24 hours and generated the syn-diastereomer as the major
Table 1 Initial Solvent Screen

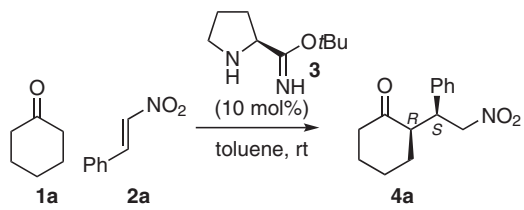

\begin{tabular}{|c|c|c|c|c|}
\hline Entry & Solvent & Conv. ${ }^{a}(\%)$ & syn/anti ratio ${ }^{\mathrm{b}}$ & ee $(s y n)^{c}(\%)$ \\
\hline 1 & DMF & 14 & 1:trace & n.d. ${ }^{d}$ \\
\hline 2 & DMSO & 0 & - & - \\
\hline 3 & 1,4-dioxane & 63 & $7: 1$ & 29 \\
\hline 4 & $\mathrm{MeCN}$ & 92 & $8: 1$ & 7 \\
\hline 5 & THF & 99 & $8: 1$ & 19 \\
\hline 6 & cyclohexane & 100 & 10.7:1 & 39 \\
\hline 7 & EtOAc & 100 & $9: 1$ & 22 \\
\hline 8 & $\mathrm{CH}_{2} \mathrm{Cl}_{2}$ & 100 & $12.6: 1$ & 21 \\
\hline 9 & diethyl carbonate & 88 & $5.6: 1$ & 28 \\
\hline 10 & toluene & $100^{\mathrm{e}}$ & $9.7: 1$ & 43 \\
\hline 11 & $\mathrm{MeOH}$ & 11 & $6.6: 1$ & n.d. ${ }^{d}$ \\
\hline 12 & MeOH-IPA (1:1) & 6 & $6: 1$ & n.d. ${ }^{d}$ \\
\hline 13 & EtOH-IPA (1:1) & 14 & $5.8: 1$ & n.d. ${ }^{d}$ \\
\hline
\end{tabular}

a Determined by $400 \mathrm{MHz}{ }^{1} \mathrm{H}$ NMR after $24 \mathrm{~h}$.

b Determined by $400 \mathrm{MHz}^{1} \mathrm{H}$ NMR.

c Determined by HPLC on a Chiralpak AS-H column (see Supporting Information).

d n.d. = not determined.

e The conversion was only $49 \%$ after $8 \mathrm{~h}$, as determined by $400 \mathrm{MHz}{ }^{1} \mathrm{H}$ NMR analysis.

product (5.8:1 dr) in 26\% ee. All other ketones showed low levels of conversion and only modest levels of enantioselectivity, with $\mathrm{N}$-Boc-piperidine-4-one (1d) and pentan-2-one (1g) not reacting at all. Although these reactions did not work well, they did provide the impetus to investigate ways to improve both the conversion and level of enantioinduction.

Benzoic acid has been reported to be a beneficial additive in the Michael reaction of ketones and nitroalkenes, ${ }^{16}$ so it was decided to investigate its effect on the reaction of tetrahydrothiopyran-4-one (1c) with $\beta$-nitrostyrene (2a). This reaction was chosen to determine the effect of benzoic acid on the conversion, the diastereoselectivity, and the enantioselectivity (Table 2). As can be seen, the introduction of benzoic acid had a dramatic and beneficial effect on the conversion and enantioselectivity of the reaction. The addition of just $10 \mathrm{~mol} \%$ of benzoic acid (Table 2, entry 1 ) led to complete consumption of the nitroalkene and generated the syn-adduct in $71 \%$ ee, a vast improvement over the $30 \%$ conversion with $32 \%$ ee from the reaction without benzoic acid. The absolute stereochemistry $(1 R, 2 S)$ of the major (syn) diastereomer was confirmed by comparison of the specific rotation and HPLC retention time with literature 


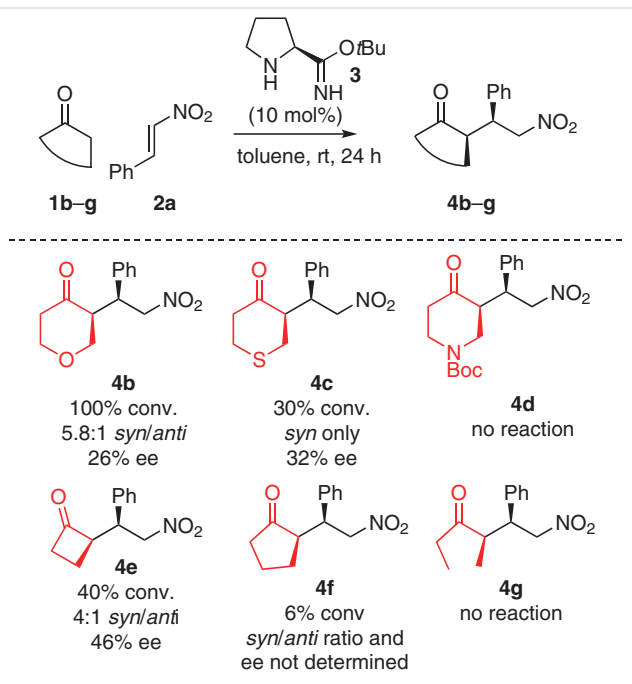

Scheme 2 Ketone scope. The conversion and syn/anti ratios were determined by $400 \mathrm{MHz}{ }^{1} \mathrm{H}$ NMR after 24 hours. The ee was determined by chiral-stationary-phase HPLC (see Supporting Information).

data. ${ }^{15}$ The diastereomeric ratio was eroded slightly, with the reaction now producing an 8:1 mixture of the syn and anti diastereomers. Increasing the amount of benzoic acid further resulted in a gradual reduction in the amount of product produced, presumably as a greater proportion of the pyrrolidine nitrogen in the catalyst was protonated and hence no longer able to participate in the reaction (entries $2-4$ ). We propose that benzoic acid serves two roles that account for its beneficial effects on the reaction. The first is that at low concentrations it aids the formation of the enamine nucleophile by protonation of the carbonyl group. The second is that it can activate the nitroalkene by simultaneously hydrogen bonding to the nitro group and the imidate in the transition state of the Michael addition, which could also account for the increase in the enantioselectivity of the reaction (Figure 1 ).

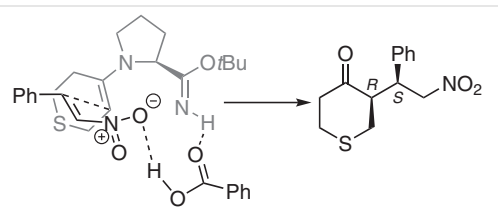

Figure 1 Proposed transition state and the role of benzoic acid

With the beneficial effect of benzoic acid verified, the reactions with substrates $\mathbf{1 a}-\mathbf{h}$ were reexamined (Scheme 3 ). In the case of cyclohexanone (1a), the ee of $\mathbf{4 a}$ increased from $43 \%$ to $60 \%$, and for the reaction of ketone $1 \mathbf{b}$, the ee of $\mathbf{4 b}$ increased from $26 \%$ to $61 \%$. Interestingly, in the case of the ketone $\mathrm{N}$-Boc-piperidine-4-one (1d), which did not react under the initial conditions, the reaction went to com-
Table 2 The Effect of Benzoic Acid on the Reaction of 1c with 2a

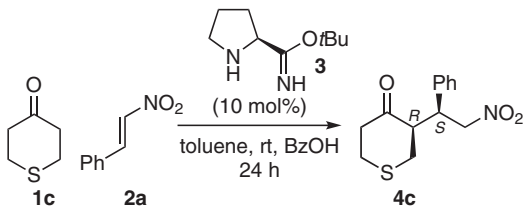

\begin{tabular}{lllll}
\hline Entry & BzOH (mol\%) & Conv. $^{\text {a }}(\%)$ & syn:anti ratio $^{\mathrm{b}}$ & ee $\left(\right.$ syn) $(\%)^{\mathrm{c}}$ \\
\hline 1 & 10 & 100 & $8: 1$ & 71 \\
2 & 50 & 90 & $7.5: 1$ & 72 \\
3 & 100 & 59 & $6: 1$ & 66 \\
4 & 150 & 49 & $8.6: 1$ & 80 \\
\hline
\end{tabular}

a Determined by $400 \mathrm{MHz}{ }^{1} \mathrm{H}$ NMR after $24 \mathrm{~h}$.

b Determined by $400 \mathrm{MHz}{ }^{1} \mathrm{H}$ NMR spectroscopy.

c Determined by chiral-stationary-phase HPLC (see Supporting Informa-

tion).

pletion and generated $\mathbf{4 d}$ as the major product in $53 \%$ ee. Under the benzoic acid conditions, $\mathbf{4 f}$ was now formed with a moderate $26 \%$ conversion and in $60 \%$ ee. Only the reactions to generate substrates $\mathbf{4 e}$ and $\mathbf{4 g}$ were unimproved by the addition of benzoic acid. In a further extension of the scope of the reaction, propanal $(\mathbf{1} \mathbf{h})$ was condensed with $\mathbf{2 a}$ to give $\mathbf{4 h}$ as the major product (14.8:1 syn/anti ratio) in $84 \%$ ee and $86 \%$ conversion. Unfortunately, the use of other functionalized aldehydes, such as (benzyloxy)acetaldehyde (1i), did not result in any reaction.

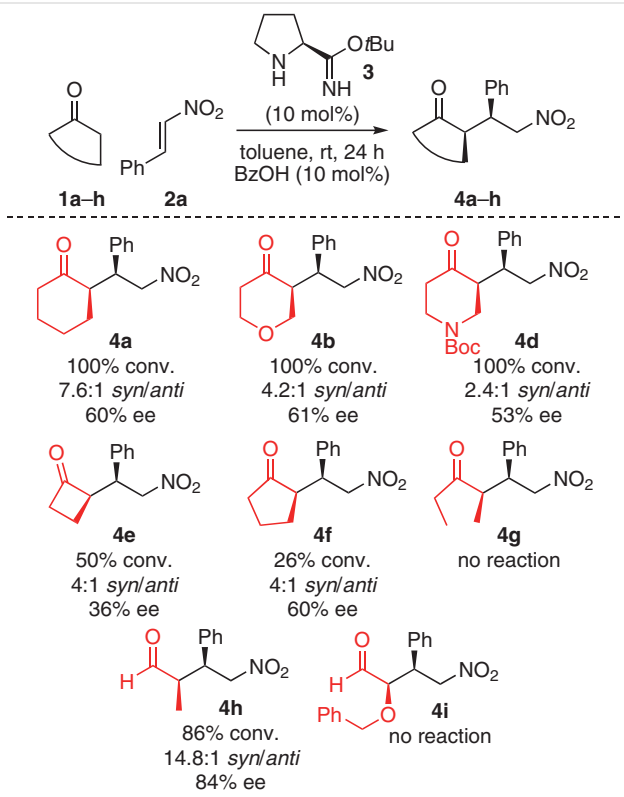

Scheme 3 Ketone and aldehyde scope with benzoic acid as an additive. The conversion and syn/anti ratios were determined by $400 \mathrm{MHz}$ ${ }^{1} \mathrm{H}$ NMR spectroscopy after 24 hours. The ee was determined by chiralstationary-phase HPLC (see Supporting Information). 
With the scope and limitations of the ketone substrate investigated, our attention turned to a study on the scope of the nitroalkene partner. To this end $\mathbf{1 a}, \mathbf{1 e}$, and $\mathbf{1 h}$ were reacted with nitroalkenes $\mathbf{2 b}-\mathbf{d}$ (Scheme 4).

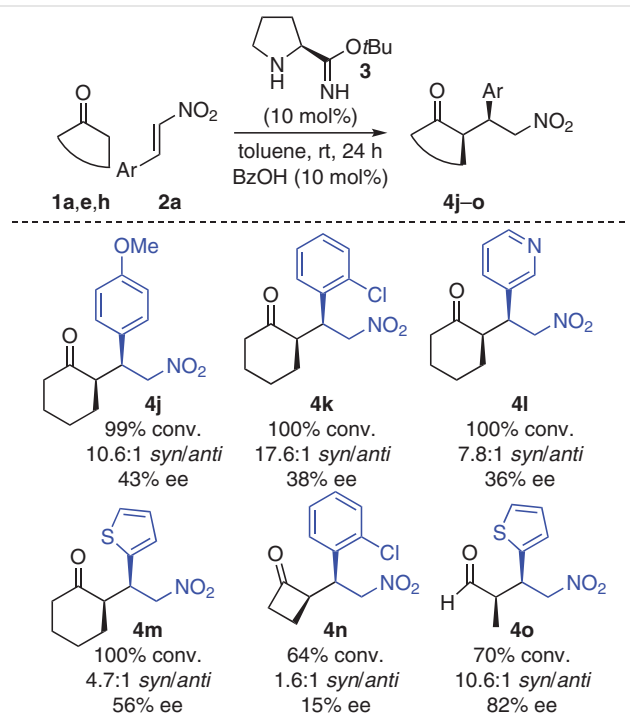

Scheme 4 Nitroalkene scope. The conversion and syn/anti ratios were determined by $400 \mathrm{MHz}{ }^{1} \mathrm{H}$ NMR after 24 hours. The ee was determined by chiral-stationary-phase HPLC (see Supporting Information).

All the nitroalkenes used in the reaction gave enantioenriched products. Both electron-rich $\mathbf{4 j}$ and electron-deficient $\mathbf{4 1}$ were produced quantitatively and in moderate ee. Two ortho-substituted aryl nitroalkenes also participated well, giving products $\mathbf{4 k}$ and $\mathbf{4 n}$. Both electron-deficient and electron-rich heteroaromatic nitroalkenes could be used in the reaction to generate products $\mathbf{4 1}, \mathbf{4 m}$, and $\mathbf{4 0}$. Pleasingly, 40 was formed in a good $82 \%$ ee.

In summary, an investigation into the use of tert-butyl proline imidate (3) as a catalyst in the Michael reactions of ketones and aldehydes with nitroalkenes was undertaken. These studies showed that imidate catalyst 3 on its own generated moderate amounts of Michael addition products in low to moderate enantioselectivities. However, the catalyst was much more efficient when combined with $10 \mathrm{~mol} \%$ of benzoic acid. Under these conditions, more product was formed and the enantioselectivity increased markedly, in some cases up to $84 \%$. The reaction was tolerant of a range of cyclic ketones, heteroatom-containing cyclic ketones, and simple aldehydes, with six-membered cyclic ketones and propanal being optimal. A range of electron-rich, electrondeficient, ortho-substituted, and heterocyclic nitroalkenes were investigated, and it was shown that they could all be used with equal efficiency. Imidates based on proline are a new class of organocatalyst that might have the potential to become efficient and selective catalysts for a range of trans- formations. Investigations into the effects of modification of the proline imidate on catalytic activity and enantioselectivity are underway and will be reported in due course.

Unless otherwise noted all compounds were bought from commercial suppliers and used without further purification. Nuclear magnetic resonance spectra were recorded on a Jeol ECS-400 spectrometer at ambient temperature; chemical shifts are quoted in parts per million (ppm) and are referenced as follows: chloroform- $d_{1}, 7.26 \mathrm{ppm}$ for ${ }^{1} \mathrm{H}$ NMR; chloroform- $d_{1}, 77.0 \mathrm{ppm}$ for ${ }^{13} \mathrm{C}$ NMR. Coupling constants $(J)$ are quoted in Hertz. Infrared spectra were recorded on a PerkinElmer UATR Two FTIR spectrometer using $\mathrm{NaCl}$ plates. Mass spectrometry was performed by the University of York mass spectrometry service using the electron-spray ionization (ESI) technique. Thin-layer chromatography was performed on aluminum sheets coated with Merck Silica gel 60 F254. The plates were developed by using ultraviolet light, basic aqueous potassium permanganate, or ethanolic anisaldehyde. Liquid chromatography was performed using forced flow (flash column) with the solvent systems indicated. The stationary phase was silica gel 60 (220-240 mesh) supplied by Sigma-Aldrich. Dry solvents were acquired from a PureSolv PS-MD7 solvent tower. Highperformance liquid chromatography (HPLC) was performed using an Agilent 1200 series instrument with the chiral columns indicated and a range of wavelengths from 210 to $280 \mathrm{~nm}$ for detection.

\section{$\boldsymbol{N}$-Boc-L-Prolinamide}

A flask was charged with Boc-L-proline $(5.01 \mathrm{~g}, 23.3 \mathrm{mmol})$ and THF ( $70 \mathrm{~mL}$ ). $\mathrm{Et}_{3} \mathrm{~N}$ (3.25 mL, $23.3 \mathrm{mmol}$ ) was added and the mixture was stirred at rt. After $15 \mathrm{~min}$, ethyl chloroformate ( $2.22 \mathrm{~mL}, 23.3 \mathrm{mmol}$ ) was added and the mixture was stirred at rt. After $1 \mathrm{~h}$, a $7 \mathrm{~N}$ solution of $\mathrm{NH}_{3}$ in $\mathrm{MeOH}(5 \mathrm{~mL}$ ) was added, and the mixture was stirred overnight until the reaction was complete $\left({ }^{1} \mathrm{H}\right.$ NMR). The solvent was removed in vacuo and the solution was washed with $\mathrm{H}_{2} \mathrm{O}(10 \mathrm{~mL})$ and extracted with $\mathrm{CH}_{2} \mathrm{Cl}_{2}(\times 3)$. The combined organic layers were dried $\left(\mathrm{MgSO}_{4}\right)$, filtered, and concentrated in vacuo to give a white solid; yield: $4.11 \mathrm{~g}$ (82\%, $19.2 \mathrm{mmol})$; mp $107-108{ }^{\circ} \mathrm{C}$ (Lit. ${ }^{19} 102-104{ }^{\circ} \mathrm{C}$ ); $[\alpha]_{D}{ }^{25}$ (deg cm $\left.\mathrm{g} \mathrm{g}^{-1} \mathrm{dm}^{-1}\right)-44.7$ (c $1.0 \mathrm{~g} \mathrm{~cm}^{-3}$, MeOH) (Lit. $\left.{ }^{14}-42.4\right)$.

IR (ATR): 3344 ( $\mathrm{N}-\mathrm{H}$ stretch), 1676 (C=O stretch), 1164 (C-O stretch) $\mathrm{cm}^{-1}$.

${ }^{1} \mathrm{H} \mathrm{NMR}\left(400 \mathrm{MHz}, \mathrm{CDCl}_{3}\right): \delta=6.85(\mathrm{~s}, 1 \mathrm{H}), 5.40-6.10(\mathrm{~m}, 1 \mathrm{H}), 4.35-$ $4.15(\mathrm{~m}, 1 \mathrm{H}), 3.55-3.25(\mathrm{~m}, 2 \mathrm{H}), 2.40-1.80(\mathrm{~m}, 4 \mathrm{H}), 1.45$ (s, $9 \mathrm{H})$.

HRMS (ESI): $m / z$ [M + Na] $]^{+}$calcd for $\mathrm{C}_{10} \mathrm{H}_{18} \mathrm{~N}_{2} \mathrm{NaO}_{3}$ : 237.1210; found 237.1209. The data agree with those reported in the literature. ${ }^{14}$

\section{N-Boc-L-Pyrrolidine-2-carbonitrile}

A flask containing $N$-Boc-L-prolinamide $(4.02 \mathrm{~g}, 18.8 \mathrm{mmol})$ and $\mathrm{Et}_{3} \mathrm{~N}$ $(5.78 \mathrm{~mL}, 41.4 \mathrm{mmol})$ in THF $(60 \mathrm{~mL})$ was cooled to $0{ }^{\circ} \mathrm{C}$, and the mixture was stirred for $30 \mathrm{~min}$. TFAA ( $3.92 \mathrm{~mL}, 28.2 \mathrm{mmol}$ ) was added, and the mixture was stirred at $0{ }^{\circ} \mathrm{C}$ for $2 \mathrm{~h}$. The mixture was then warmed to rt and stirred overnight until the reaction was complete (TLC: $100 \%$ EtOAc; CAM stain). The solvent was removed in vacuo and the crude yellow oil was redissolved in EtOAc, washed with $2 \mathrm{M} \mathrm{HCl}$, and extracted with EtOAc ( $\times 3)$. The organic layers were combined, washed with sat. aq $\mathrm{NaHCO}_{3}$ and brine. The organic layers were combined, dried $\left(\mathrm{Na}_{2} \mathrm{SO}_{4}\right)$, and filtered. The solution was concentrated in vacuo to give the crude product as an orange oil. The crude oil was further purified by column chromatography (silica gel, gradient 
hexane-EtOAc) to give a pale-yellow oil; yield: $3.51 \mathrm{~g}(95 \%, 17.9$ $\mathrm{mmol}$ ); $[\alpha]_{D}{ }^{20}-72.77$ (c $\left.1.0 \mathrm{mg} / \mathrm{mL}, \mathrm{MeOH}\right)\left\{\right.$ Lit. $^{14}[\alpha]_{D}{ }^{20}-91.15$ (c 1.3 $\mathrm{mg} / \mathrm{mL}, \mathrm{MeOH})\}$.

IR (ATR): 2980, 1694, 1387, $1158 \mathrm{~cm}^{-1}$.

$\left.{ }^{1} \mathrm{H} \mathrm{NMR} \mathrm{(400} \mathrm{MHz,} \mathrm{CDCl}_{3}\right): \delta=4.60-4.40(\mathrm{~m}, 1 \mathrm{H}), 3.58-3.25(\mathrm{~m}, 2 \mathrm{H})$, 2.30-1.95 (m, $4 \mathrm{H}), 1.50-1.45(\mathrm{~m}, 9 \mathrm{H})$.

${ }^{13} \mathrm{C}$ NMR (101 $\mathrm{MHz}, \mathrm{CDCl}_{3}$ ): $\delta=153.8$ and 153.2 (rotamers), 119.2, 81.6 and 81.1 (rotamers), 47.3 and 47.1 (rotamers), 46.1 and 45.8 (rotamers), 31.7 and 30.9 (rotamers), 28.4 and 28.3 (rotamers), 24.7 and 23.9 (rotamers).

HRMS (ESI): $m / z[\mathrm{M}+\mathrm{Na}]^{+}$calcd for $\mathrm{C}_{10} \mathrm{H}_{16} \mathrm{~N}_{2} \mathrm{NaO}_{2}: 219.1104$; found: 219.1102. The data agree with those reported in the literature. ${ }^{14}$

\section{tert-Butyl L-Proline Imidate Trifluoroacetic Acid Salt}

TFA (17.00 mL, $229.5 \mathrm{mmol}$ ) was added to a flask containing $N$-Boc-Lpyrrolidine-2-carbonitrile $(1.0 \mathrm{~g}, 5.1 \mathrm{mmol})$, and the flask was cooled to $0{ }^{\circ} \mathrm{C}$. Upon consumption of the starting material (TLC; hexane-EtOAc, 8:2; CAM stain), $t$-BuOH $(0.97 \mathrm{~mL}, 10.2 \mathrm{mmol})$ was added and the mixture was allowed to warm to rt and stirred overnight. Stirring was then stopped and the solvent was removed in vacuo. Trituration with diisopropyl ether-hexane gave the salt as a yellow solid; yield: $1.1 \mathrm{~g}$ (77\%, $3.9 \mathrm{mmol}$ ); $\mathrm{mp} 87-89{ }^{\circ} \mathrm{C}$ (Lit. ${ }^{14} 88-90{ }^{\circ} \mathrm{C}$ ). $[\alpha]_{\mathrm{D}}{ }^{20}-44.36$ (c 1.0 $\mathrm{mg} / \mathrm{mL}, \mathrm{CH}_{2} \mathrm{Cl}_{2}$ ); Lit. ${ }^{14}[\alpha]_{\mathrm{D}}{ }^{25}-47.23\left(c 1.0 \mathrm{mg} / \mathrm{mL}, \mathrm{CH}_{2} \mathrm{Cl}_{2}\right.$ ).

IR (ATR): 1661, 1177, $1131 \mathrm{~cm}^{-1}$.

${ }^{1} \mathrm{H}$ NMR (400 MHz, $\left.\mathrm{CD}_{3} \mathrm{OD}\right): \delta=4.12(\mathrm{dd}, J=8.5,6.8 \mathrm{~Hz}, 1 \mathrm{H}), 3.42-$ $3.33(\mathrm{~m}, 1 \mathrm{H}), 3.36-3.24(\mathrm{~m}, 1 \mathrm{H}), 2.43-2.31(\mathrm{~m}, 1 \mathrm{H}), 2.06-1.85$ (m, 3 $\mathrm{H}), 1.33(\mathrm{~s}, 9 \mathrm{H})$.

${ }^{13} \mathrm{C}$ NMR (101 MHz, $\left.\mathrm{CDCl}_{3}\right): \delta=167.7,167.6,59.7,52.2,52.1,46.4$, 30.5, 28.5, 24.7 (TFA signals are absent).

HRMS (ESI): $m / z[M+H]^{+}$calcd for $\mathrm{C}_{9} \mathrm{H}_{19} \mathrm{~N}_{2} \mathrm{O}$ : 171.1492; found: 171.1493. The data agree with those in the literature. ${ }^{14}$

\section{tert-Butyl L-Proline Imidate (3)}

The freet-proline imidate 3 was liberated by dissolving the salt ( $1.0 \mathrm{~g}$, $3.9 \mathrm{mmol}$ ) in $\mathrm{CH}_{2} \mathrm{Cl}_{2}$ and stirring over $\mathrm{K}_{2} \mathrm{CO}_{3}(2.69 \mathrm{~g}, 19.5 \mathrm{mmol})$ for 1 $\mathrm{h}$ before filtering and concentrating in vacuo. The crude product was purified by column chromatography [silica gel, gradient $\mathrm{CH}_{2} \mathrm{Cl}_{2}$ $\mathrm{MeOH}$; TLC $\left(\mathrm{CH}_{2} \mathrm{Cl}_{2}-\mathrm{MeOH} 8: 2\right.$, CAM stain)] to give a yellow solid; yield: $0.4 \mathrm{~g}(62 \%, 2.4 \mathrm{mmol})$; $\mathrm{mp} 68-69^{\circ} \mathrm{C}$. $[\alpha]_{\mathrm{D}}{ }^{20}-51.54($ c $1.0 \mathrm{mg} / \mathrm{mL}$, $\mathrm{MeOH})$.

IR (ATR): 2965, 1657, 1518, 1454, $1226 \mathrm{~cm}^{-1}$.

${ }^{1} \mathrm{H} \mathrm{NMR}\left(400 \mathrm{MHz}, \mathrm{CDCl}_{3}\right): \delta=7.48-7.40(\mathrm{br} \mathrm{s}, 1 \mathrm{H}), 3.60(\mathrm{dd}, J=8.9$, $5.5 \mathrm{~Hz}, 1 \mathrm{H}), 2.98(\mathrm{dt}, J=10.3,6.8 \mathrm{~Hz}, 1 \mathrm{H}), 2.86(\mathrm{dt}, J=10.3,6.4 \mathrm{~Hz}, 1$ H), 2.26 (br s, $1 \mathrm{H}$ ), 2.12-2.01 (m, $1 \mathrm{H}), 1.91-1.79$ (m, $1 \mathrm{H}), 1.77-1.54$ (m, $2 \mathrm{H}), 1.32(\mathrm{~s}, 9 \mathrm{H})$.

${ }^{13} \mathrm{C}$ NMR $\left(101 \mathrm{MHz}, \mathrm{CDCl}_{3}\right): \delta=174.3,61.2,50.2,47.3,30.8,28.8,26.3$. HRMS (ESI): $m / z[\mathrm{M}+\mathrm{H}]^{+}$calcd for $\mathrm{C}_{9} \mathrm{H}_{19} \mathrm{~N}_{2} \mathrm{O}$ : 171.1492; found: 171.1492. The data agree with those reported in the literature. ${ }^{14}$

\section{Michael Reactions Catalyzed by Proline Imidate 3; General Proce- dure}

A flame-dried flask under a $\mathrm{N}_{2}$ atmosphere was charged with the proline imidate 3 ( $0.025 \mathrm{mmol}, 0.1$ equiv), benzoic acid ( $0.025 \mathrm{mmol}, 0.1$ equiv), and the appropriate ketone $\mathbf{1}(1.250 \mathrm{mmol}, 5$ equiv) in toluene $(1 \mathrm{~mL})$, and the mixture and stirred for $15 \mathrm{~min}$. Nitroalkene $\mathbf{2}(0.250$ mmol, 1.0 equiv) was then added and the mixture was stirred at $\mathrm{rt}$ for $24 \mathrm{~h}$. The reaction was then quenched with sat. aq $\mathrm{NH}_{4} \mathrm{Cl}(2 \mathrm{~mL})$ and the mixture was extracted with $\mathrm{CH}_{2} \mathrm{Cl}_{2}(\times 3)$. The organic layers were collected, washed with $0.7 \mathrm{M}$ aq $\mathrm{K}_{2} \mathrm{CO}_{3}$, dried $\left(\mathrm{Na}_{2} \mathrm{SO}_{4}\right)$, filtered, and concentrated to give the crude Michael product.

\section{(2R)-2-[(1S)-2-Nitro-1-phenylethyl]cyclohexanone (4a)}

Flash column chromatography (silica gel, gradient hexane- $\mathrm{Et}_{2} \mathrm{O}$ ); isolated yield: $81 \%$ (49.8 $\mathrm{mg}$ from $37.3 \mathrm{mg}$ of alkene); white solid; $\mathrm{mp}$ 118-120 ${ }^{\circ} \mathrm{C}$ (Lit. ${ }^{11 \mathrm{c}} 128-130{ }^{\circ} \mathrm{C}$ ); syn/anti: $27.5: 1.0$; ee (syn): $60 \%$ (by chiral HPLC); $[\alpha]_{D}{ }^{20}+14.4\left(c 0.58 \mathrm{mg} / \mathrm{mL}, \mathrm{CHCl}_{3}\right)\left\{\right.$ Lit. $[\alpha]_{D}{ }^{25}+19.1$ (c $\left.\left.1.0 \mathrm{mg} / \mathrm{mL}, \mathrm{CHCl}_{3}\right)\right\} .{ }^{11 \mathrm{c}}$

HPLC: CHIRALPAK AS-H [i-PrOH-hexane (25:75), flow rate: $\left.1 \mathrm{~mL} / \mathrm{min}, \lambda=254 \mathrm{~nm}, 30^{\circ} \mathrm{C}\right]$.

IR (ATR): 2977.28, 1706.60, 1550.44, 1379.83, 1130.14, $702.19 \mathrm{~cm}^{-1}$. $\left.{ }^{1} \mathrm{H} \mathrm{NMR} \mathrm{(400} \mathrm{MHz,} \mathrm{CDCl}_{3}\right)($ syn $): \delta=7.39-7.22(\mathrm{~m}, 3 \mathrm{H}), 7.22-7.08(\mathrm{~m}$, $2 \mathrm{H}), 4.93$ (dd, $J=12.6,4.5 \mathrm{~Hz}, 1 \mathrm{H}), 4.62(\mathrm{dd}, J=12.6,9.9 \mathrm{~Hz}, 1 \mathrm{H}), 3.75$ (td, $J=10.0,4.5 \mathrm{~Hz}, 1 \mathrm{H}), 2.73-2.62(\mathrm{~m}, 1 \mathrm{H}), 2.52-2.25$ (m, $2 \mathrm{H}), 2.13-$ $2.01(\mathrm{~m}, 1 \mathrm{H}), 1.83-1.48(\mathrm{~m}, 4 \mathrm{H}), 1.31-1.15(\mathrm{~m}, 1 \mathrm{H})$. The data agree with those reported in the literature. ${ }^{15 a}$ Detected anti-isomer signal: $\delta$ $=4.02-3.97(\mathrm{~m}, 1 \mathrm{H}) .^{15 \mathrm{~b}}$

${ }^{13} \mathrm{C}$ NMR $\left(101 \mathrm{MHz}, \mathrm{CDCl}_{3}\right)($ syn $): \delta=212.1,135.9,129.1,128.3,127.9$, 79.0, 52.6, 44.02, 42.9, 33.3, 28.6, 25.1.

HRMS (ESI): $m / z$ [M $+\mathrm{Na}]^{+}$calcd for $\mathrm{C}_{14} \mathrm{H}_{17} \mathrm{NNaO}_{3}: 270.1101$; found: 270.1103.

\section{(2R)-2-[(1S)-2-Nitro-1-phenylethyl]tetrahydropyran-4-one (4b)}

Flash column chromatography (silica gel, gradient hexane-Et ${ }_{2} \mathrm{O}$ ); isolated yield: $87 \%$ (54.3 mg from $37.3 \mathrm{mg}$ of alkene); white solid; syn/anti: 10.1:1.0; ee (syn): $61 \%$ (by chiral HPLC).

HPLC: CHIRALPAK IA [ $i$-PrOH-hexane (15:85), flow rate: $1 \mathrm{~mL} / \mathrm{min}, \lambda$ $=210 \mathrm{~nm}, 25^{\circ} \mathrm{C}$.

IR (ATR): 2977.27, 2831.30, 1711.09, 1551.61, 1380.25, $702.69 \mathrm{~cm}^{-1}$. ${ }^{1} \mathrm{H} \mathrm{NMR}\left(400 \mathrm{MHz}, \mathrm{CDCl}_{3}\right.$ ) (syn): $\delta=7.37-7.26(\mathrm{~m}, 3 \mathrm{H}), 7.19-7.14(\mathrm{~m}$, $2 \mathrm{H}), 4.92(\mathrm{dd}, J=12.7,4.6 \mathrm{~Hz}, 1 \mathrm{H}), 4.63(\mathrm{dd}, J=12.7,10.1 \mathrm{~Hz}, 1 \mathrm{H})$, 4.18-4.09 (m, $1 \mathrm{H}), 3.87-3.62(\mathrm{~m}, 3 \mathrm{H}), 3.26(\mathrm{dd}, J=11.6,8.9 \mathrm{~Hz}, 1 \mathrm{H})$, 2.92-2.82 (m, $1 \mathrm{H}), 2.71-2.61(\mathrm{~m}, 1 \mathrm{H}), 2.55(\mathrm{dt}, J=13.9,4.0 \mathrm{~Hz}, 1 \mathrm{H})$. The data agree with those reported in the literature. ${ }^{15}$ Detected antiisomer signals: $\delta=4.89-4.83(\mathrm{~m}, 1 \mathrm{H}), 3.95(\mathrm{dt}, J=8.9,6.0 \mathrm{~Hz}, 1 \mathrm{H})$, 3.52-3.45 (m, $1 \mathrm{H}), 2.97(\mathrm{dt}, J=8.6,5.6 \mathrm{~Hz}, 1 \mathrm{H}), 2.52-2.43(\mathrm{~m}, 1 \mathrm{H})$.

${ }^{13} \mathrm{C}$ NMR $\left(101 \mathrm{MHz}, \mathrm{CDCl}_{3}\right)($ syn $): \delta=207.5,136.3,129.4,128.4,128.0$, 78.8, 71.7, 69.1, 53.4, 43.1, 41.4 .

HRMS (ESI): $m / z$ [M + Na] $]^{+}$calcd for $\mathrm{C}_{13} \mathrm{H}_{15} \mathrm{NNaO}_{4}: 272.0893$; found: $\mathrm{C}_{13} \mathrm{H}_{15} \mathrm{NNaO}_{4}$ : 272.0893 .

\section{(2S)-2-[(1S)-2-Nitro-1-phenylethyl]tetrahydrothiopyran-4-one (4c)}

Flash column chromatography ( silica gel, gradient hexane- $\mathrm{Et}_{2} \mathrm{O}$ ); isolated yield: $75 \%$ (49.9 mg from $37.3 \mathrm{mg}$ of alkene); white solid; syn/anti: 7.8:1.0; ee (syn): 71\% (by chiral HPLC).

HPLC: CHIRALPAK IA [i-PrOH-hexane (15:85), flow rate: 0.95 $\mathrm{mL} / \mathrm{min}, \lambda=210 \mathrm{~nm}, 25^{\circ} \mathrm{C}$.

IR (ATR): 2970.77, 2916.50, 1705.81, 1549.81, 1549.56, 1379.93, $701.61 \mathrm{~cm}^{-1}$.

${ }^{1} \mathrm{H} \mathrm{NMR}\left(400 \mathrm{MHz}, \mathrm{CDCl}_{3}\right.$ ) (syn): $\delta=7.38-7.26(\mathrm{~m}, 3 \mathrm{H}), 7.20-7.15(\mathrm{~m}$, $2 \mathrm{H}), 4.73$ (dd, $J=12.8,4.6 \mathrm{~Hz}, 1 \mathrm{H}), 4.62(\mathrm{dd}, J=12.8,9.8 \mathrm{~Hz}, 1 \mathrm{H}), 3.97$ (td, $J=10.2,4.6 \mathrm{~Hz}, 1 \mathrm{H}), 3.08-3.00(\mathrm{~m}, 1 \mathrm{H}), 3.00-2.92(\mathrm{~m}, 2 \mathrm{H}), 2.92-$ 2.75 (m, 2 H), 2.60 (ddd, $J=13.9,4.1,1.8 \mathrm{~Hz}, 1 \mathrm{H}$ ), 2.44 (dd, $J=13.9,9.4$ 
$\mathrm{Hz}, 1 \mathrm{H}$ ). Data (syn) agree with those reported in the literature. ${ }^{15}$ Detected anti-isomer signals: $\delta=4.92-4.77(\mathrm{~m}, 2 \mathrm{H}), 4.18-4.11(\mathrm{~m}, 1 \mathrm{H})$, 3.15-3.09 (m, $1 \mathrm{H})$.

${ }^{13} \mathrm{C}$ NMR $\left(101 \mathrm{MHz}, \mathrm{CDCl}_{3}\right)(\mathrm{syn}): \delta=209.6,136.6,129.4,128.4,128.3$, 78.7, 55.1, 44.6, 43.6, 35.2, 31.7.

HRMS (ESI): $m / z$ [M + Na] ${ }^{+}$calcd for $\mathrm{C}_{13} \mathrm{H}_{15} \mathrm{NNaO}_{3} \mathrm{~S}: 288.0665$; found: $\mathrm{C}_{13} \mathrm{H}_{15} \mathrm{NNaO}_{3} \mathrm{~S}: 288.0669$.

\section{tert-Butyl (2S)-2-(2-Nitro-1-phenylethyl)-4-oxopiperidine-1-car- boxylate (4d)}

Flash column chromatography ( silica gel, gradient hexane- $\mathrm{Et}_{2} \mathrm{O}$ ); isolated yield: $71 \%$ (61.8 mg from $37.3 \mathrm{mg}$ of alkene); white solid; syn/anti: 3.8:1.0; ee (syn): 53\% (chiral HPLC).

HPLC: CHIRALPAK IC [ $i$-PrOH-hexane (10:90), flow rate: $1.3 \mathrm{~mL} / \mathrm{min}$, $\left.\lambda=210 \mathrm{~nm}, 25^{\circ} \mathrm{C}\right]$

IR (ATR): 2976.97, 2927.85, 1689.38, 1551.11, 1420.82, 1366.43, 1239.72, 1160.53, 731.30, $701.08 \mathrm{~cm}^{-1}$.

${ }^{1} \mathrm{H}$ NMR $\left(400 \mathrm{MHz}, \mathrm{CDCl}_{3}\right)$ (syn): $\delta=7.36-7.22(\mathrm{~m}, 3 \mathrm{H}), 7.22-7.14(\mathrm{~m}$, $2 \mathrm{H}), 4.91$ (dd, $J=12.7,4.6 \mathrm{~Hz}, 1 \mathrm{H}), 4.59(\mathrm{dd}, J=12.7,9.8 \mathrm{~Hz}, 1 \mathrm{H}), 4.19$ (br s, $1 \mathrm{H}$ ), 3.81 (br s, $2 \mathrm{H}$ ), 3.29-3.05 (m, $1 \mathrm{H}), 2.88-2.60(\mathrm{~m}, 2 \mathrm{H}$ ), 2.57-2.39 (m, $2 \mathrm{H}), 1.60-1.08(\mathrm{~m}, 9 \mathrm{H})$. The data agree with those reported in the literature. ${ }^{15}$ Detected anti-isomer signals: $\delta=4.96-4.82$ ( $\mathrm{m}, 1 \mathrm{H}), 3.45-3.33(\mathrm{~m}, 1 \mathrm{H}), 3.29-3.05(\mathrm{~m}, 1 \mathrm{H}), 2.35(\mathrm{t}, J=6.4 \mathrm{~Hz}, 2$ $\mathrm{H})$.

${ }^{13} \mathrm{C}$ NMR (101 MHz, $\mathrm{CDCl}_{3}$ ) (syn): $\delta=208.5,154.2,136.6,129.3,129.1$, 128.3, 128.2, 128.1, 80.8, 79.0, 44.3, 41.9, 41.9, 40.9, 28.3.

HRMS (ESI): $m / z[\mathrm{M}+\mathrm{Na}]^{+}$calcd for $\mathrm{C}_{18} \mathrm{H}_{24} \mathrm{~N}_{2} \mathrm{NaO}_{5}$ : 371.1577; found: $\mathrm{C}_{18} \mathrm{H}_{24} \mathrm{~N}_{2} \mathrm{NaO}_{5}$ : 371.1586 .

\section{(2R)-2-[(1S)-2-Nitro-1-phenylethyl]cyclobutanone (4e)}

Flash column chromatography ( silica gel, gradient hexane- $\mathrm{Et}_{2} \mathrm{O}$ ); isolated yield: $27 \%$ (14.8 mg from $37.3 \mathrm{mg}$ of alkene); yellow oil; syn/anti: 2.4:1.0; ee (syn): $37 \%$ ee (by chiral HPLC).

HPLC: CHIRALPAK AS-H [i-PrOH-hexane (25:75), flow rate: 0.7 $\left.\mathrm{mL} / \mathrm{min}, \lambda=210 \mathrm{~nm}, 25^{\circ} \mathrm{C}\right]$.

IR (ATR): 2923.30, 1774.51, 1550.51, 1379.41, 1086.02, $701.54 \mathrm{~cm}^{-1}$. ${ }^{1} \mathrm{H} \mathrm{NMR}\left(400 \mathrm{MHz}, \mathrm{CDCl}_{3}\right.$ ) (syn): $\delta=7.38-7.26(\mathrm{~m}, 3 \mathrm{H}), 7.21-7.15(\mathrm{~m}$, $2 \mathrm{H}), 5.06(\mathrm{dd}, J=12.8,4.6 \mathrm{~Hz}, 1 \mathrm{H}), 4.63(\mathrm{dd}, J=12.8,9.9 \mathrm{~Hz}, 1 \mathrm{H}$ ), 3.76-3.65 (m, $1 \mathrm{H}), 3.65-3.52$ (m, $1 \mathrm{H}), 3.15-2.87$ (m, $2 \mathrm{H}), 2.10-1.98$ $(\mathrm{m}, 1 \mathrm{H}), 1.78-1.60(\mathrm{~m}, 1 \mathrm{H})$. Data (syn) agree with those reported in the literature. ${ }^{11 \mathrm{~b}}$ anti-Isomer signals: $\delta=7.38-7.26(\mathrm{~m}, 3 \mathrm{H}), 7.21-$ 7.15 (m, 2 H), 4.92-4.76 (m, 2 H), 3.76-3.65 (m, 2 H), 3.15-2.87 (m, 1 $\mathrm{H}), 2.68-2.57$ (m, $1 \mathrm{H}), 2.22-2.10(\mathrm{~m}, 1 \mathrm{H}), 1.78-1.60(\mathrm{~m}, 1 \mathrm{H})$.

${ }^{13} \mathrm{C}$ NMR $\left(101 \mathrm{MHz}, \mathrm{CDCl}_{3}\right)($ syn $): \delta=208.7,137.4,129.2,128.3,127.7$, 78.3, 61.1, 44.6, 44.4, 15.9. Detected anti-isomer signals: $\delta=136.5$, 129.1, 128.3, 77.7, 61.5, 45.1, 44.3, 14.4.

HRMS (ESI): $m / z$ [M $+\mathrm{Na}]^{+}$calcd for $\mathrm{C}_{12} \mathrm{H}_{13} \mathrm{NNaO}_{3}: 242.0788$; found: $\mathrm{C}_{12} \mathrm{H}_{13} \mathrm{NNaO}_{3}$ : 242.0786 .

\section{(2R)-2-[(1S)-2-Nitro-1-phenylethyl]cyclopentanone (4f)}

Flash column chromatography ( silica gel, gradient hexane- $\mathrm{Et}_{2} \mathrm{O}$ ); isolated yield: $21 \%$ (12.2 mg from $37.3 \mathrm{mg}$ of alkene); white solid; syn/anti: 6.0:1.0; ee (syn): 60\% (by chiral HPLC).

HPLC: CHIRALPAK AS-H [i-PrOH-hexane (25:75), flow rate: 1 $\mathrm{mL} / \mathrm{min}, \lambda=210 \mathrm{~nm}, 25^{\circ} \mathrm{C}$.
IR (ATR): 2967.49, 1732.30, 1549.98, 1379.68, 1154.87, $702.35 \mathrm{~cm}^{-1}$. ${ }^{1} \mathrm{H}$ NMR $\left(400 \mathrm{MHz}, \mathrm{CDCl}_{3}\right)$ (syn): $\delta=7.36-7.21(\mathrm{~m}, 3 \mathrm{H}), 7.21-7.11(\mathrm{~m}$, $2 \mathrm{H}), 5.33(\mathrm{dd}, J=12.9,5.6 \mathrm{~Hz}, 1 \mathrm{H}), 4.70(\mathrm{dd}, J=12.9,9.8 \mathrm{~Hz}, 1 \mathrm{H}), 3.68$ $(\mathrm{td}, J=9.5,5.6 \mathrm{~Hz}, 1 \mathrm{H}), 2.45-2.29(\mathrm{~m}, 2 \mathrm{H}), 2.18-2.06(\mathrm{~m}, 1 \mathrm{H}), 1.98-$ 1.77 (m, $2 \mathrm{H}), 1.77-1.62(\mathrm{~m}, 1 \mathrm{H}), 1.54-1.39$ ( $\mathrm{m}, 1 \mathrm{H})$. Data (syn) agree with those reported in the literature. ${ }^{11 \mathrm{~b}}$ Detected anti-isomer signals: $\delta=5.01(\mathrm{~d}, J=7.8 \mathrm{~Hz}, 2 \mathrm{H}), 3.85-3.78(\mathrm{~m}, 1 \mathrm{H}), 2.54-2.46(\mathrm{~m}, 1 \mathrm{H})$, 2.29-2.22 (m, $1 \mathrm{H})$.

${ }^{13} \mathrm{C}$ NMR $\left(101 \mathrm{MHz}, \mathrm{CDCl}_{3}\right)($ syn $): \delta=218.6,137.8,129.0,128.1,128.0$, 78.4, 50.6, 44.3, 38.8, 28.4, 20.4.

HRMS (ESI): $m / z$ [M + Na] $]^{+}$calcd for $\mathrm{C}_{13} \mathrm{H}_{15} \mathrm{NNaO}_{3}$ : 256.0944; found: $\mathrm{C}_{13} \mathrm{H}_{15} \mathrm{NNaO}_{3}: 256.0942$.

\section{(2R,3S)-2-Methyl-4-nitro-3-phenylbutanal (4h)}

Flash column chromatography ( silica gel, gradient hexane- $\mathrm{Et}_{2} \mathrm{O}$ ); isolated yield: $85 \%$ (44.0 mg from $37.3 \mathrm{mg}$ of alkene); yellow oil; syn/anti: 4.6:1.0 (epimerization over time syn to anti); ee (syn): $84 \%$ (by chiral HPLC).

HPLC: CHIRALPAK IC [ $i$-PrOH-hexane (10:90), flow rate: $1.3 \mathrm{~mL} / \mathrm{min}$, $\left.\lambda=210 \mathrm{~nm}, 25^{\circ} \mathrm{C}\right]$.

IR (ATR): 2974.90, 2730.6, 1722.71, 1551.14, 1379.6, $702.48 \mathrm{~cm}^{-1}$.

${ }^{1} \mathrm{H} \mathrm{NMR}\left(400 \mathrm{MHz}, \mathrm{CDCl}_{3}\right)($ syn $): \delta=9.70(\mathrm{~d}, J=1.8 \mathrm{~Hz}, 1 \mathrm{H}), 7.36-7.25$ (m, $3 \mathrm{H}), 7.22-7.11(\mathrm{~m}, 2 \mathrm{H}), 4.84-4.71(\mathrm{~m}, 1 \mathrm{H}), 4.71-4.62(\mathrm{~m}, 1 \mathrm{H})$, $3.80(\mathrm{td}, J=9.1,5.7 \mathrm{~Hz}, 1 \mathrm{H}), 2.86-2.70(\mathrm{~m}, 1 \mathrm{H}), 0.98(\mathrm{~d}, J=7.2 \mathrm{~Hz}, 3$ $\mathrm{H})$. Data (syn) agree with those reported in the literature. ${ }^{11 \mathrm{e}}$ anti-Isomer signals: $\delta=9.52(\mathrm{~d}, J=1.8 \mathrm{~Hz}, 1 \mathrm{H}), 7.36-7.25(\mathrm{~m}, 3 \mathrm{H}), 7.22-7.11$ (m, $2 \mathrm{H}), 4.84-4.71(\mathrm{~m}, 2 \mathrm{H}), 3.80(\mathrm{td}, J=9.1,5.7 \mathrm{~Hz}, 1 \mathrm{H}), 2.86-2.70$ $(\mathrm{m}, 1 \mathrm{H}), 1.20(\mathrm{~d}, J=7.2 \mathrm{~Hz}, 2 \mathrm{H})$.

${ }^{13} \mathrm{C}$ NMR $\left(101 \mathrm{MHz}, \mathrm{CDCl}_{3}\right)($ syn $): \delta=202.39,136.64,129.19,128.27$ \& $128.24_{\text {syn/anti }}, 128.17,78.21,48.53,44.12,12.23$. Detected anti-isomer signals: $\delta=202.5,137.0,129.2,128.3 \& 128.2_{\text {syn/anti }}$, 48.8, 44.9, 11.8 .

HRMS (ESI): $m / z[\mathrm{M}+\mathrm{Na}]^{+}$calcd for $\mathrm{C}_{11} \mathrm{H}_{13} \mathrm{NNaO}_{3}$ : 230.0788; found: $\mathrm{C}_{11} \mathrm{H}_{13} \mathrm{NNaO}_{3}$ : 230.0788 .

\section{(2R)-2-[(1S)-1-(4-Methoxyphenyl)-2-nitroethyl]cyclohexanone (4j)}

Flash column chromatography ( silica gel, gradient hexane- $\mathrm{Et}_{2} \mathrm{O}$ ); isolated yield: $75 \%$ (51.9 mg from $44.8 \mathrm{mg}$ of alkene); white solid; syn/anti: 10.0:1.0; ee (syn): 43\% (by chiral HPLC).

HPLC: CHIRALPAK IA [ $i$-PrOH-hexane (10:90), flow rate: $0.5 \mathrm{~mL} / \mathrm{min}$, $\left.\lambda=254 \mathrm{~nm}, 25^{\circ} \mathrm{C}\right]$.

IR (ATR): 2940.52, 2862.98, 1706.24, 1550.11, 1514.27, 1251.39, $831.82 \mathrm{~cm}^{-1}$.

$\left.{ }^{1} \mathrm{H} \mathrm{NMR} \mathrm{(400} \mathrm{MHz,} \mathrm{CDCl}_{3}\right)($ syn $): \delta=7.11-7.02(\mathrm{~m}, 2 \mathrm{H}), 6.87-6.79(\mathrm{~m}$, $2 \mathrm{H}), 4.90$ (dd, $J=12.4,4.6 \mathrm{~Hz}, 1 \mathrm{H}), 4.57(\mathrm{dd}, J=12.4,10.0 \mathrm{~Hz}, 1 \mathrm{H})$, $3.77(\mathrm{~s}, 3 \mathrm{H}), 3.70(\mathrm{td}, J=9.9,4.6 \mathrm{~Hz}, 1 \mathrm{H}), 2.69-2.58(\mathrm{~m}, 1 \mathrm{H}), 2.51-$ 2.31 (m, $2 \mathrm{H}), 2.11-2.01$ (m, $1 \mathrm{H}), 1.82-1.47$ (m, $4 \mathrm{H}), 1.29-1.14$ (m, 1 $\mathrm{H})$. Data (syn) agree with those reported in the literature. ${ }^{11 \mathrm{~d}}$ Detected anti-isomer signals: $\delta=7.19-7.13(\mathrm{~m}, 2 \mathrm{H}), 6.86-6.81(\mathrm{~m}, 2 \mathrm{H}), 4.79$ (dd, $J=12.7,9.7 \mathrm{~Hz}, 1 \mathrm{H}$ ), 3.93-3.87 (m, $1 \mathrm{H}), 1.43-1.34(\mathrm{~m}, 1 \mathrm{H})$.

${ }^{13} \mathrm{C}$ NMR $\left(101 \mathrm{MHz}, \mathrm{CDCl}_{3}\right.$ ) (syn): $\delta=212.2,159.1,129.6,129.3,114.4$, 79.2, 55.3, 52.8, 43.3, 42.8, 33.2, 28.6, 25.1.

HRMS (ESI): $m / z[\mathrm{M}+\mathrm{Na}]^{+}$calcd for $\mathrm{C}_{15} \mathrm{H}_{19} \mathrm{NNaO}_{4}$ : 300.1206; found: $\mathrm{C}_{15} \mathrm{H}_{19} \mathrm{NNaO}_{4}: 300.1210$. 
(2R)-2-[(1S)-1-(2-Chlorophenyl)-2-nitroethyl]cyclohexanone (4k) Flash column chromatography (silica gel, gradient hexane- $\mathrm{Et}_{2} \mathrm{O}$ ); isolated yield: $96 \%$ (67.4 mg from $45.9 \mathrm{mg}$ of alkene); white solid; mp 67-69 ${ }^{\circ} \mathrm{C}$ (Lit. ${ }^{11 \mathrm{c}} 64-66^{\circ} \mathrm{C}$ ); syn/anti: 20.0:1.0; ee (syn): $38 \%$ (by chiral HPLC). $[\alpha]_{\mathrm{D}}{ }^{20}+15.30\left(\right.$ c $\left.0.58 \mathrm{mg} / \mathrm{mL}, \mathrm{CHCl}_{3}\right)\left\{\right.$ Lit. $[\alpha]_{\mathrm{D}}{ }^{25}+45.3$ (c 1.0 $\left.\left.\mathrm{mg} / \mathrm{mL}, \mathrm{CHCl}_{3}\right)\right\}{ }^{11 \mathrm{c}}$

HPLC: CHIRALPAK IA [ $i$-PrOH-hexane (10:90), flow rate: $1 \mathrm{~mL} / \mathrm{min}$, $\left.\lambda=254 \mathrm{~nm}, 25^{\circ} \mathrm{C}\right]$.

IR (ATR): 2940.72, 2862.97, 1706.27, 1550.36, 1379.07, $754.95 \mathrm{~cm}^{-1}$. ${ }^{1} \mathrm{H}$ NMR $\left(400 \mathrm{MHz}, \mathrm{CDCl}_{3}\right.$ ) (syn): $\delta=7.39-7.34(\mathrm{~m}, 1 \mathrm{H}), 7.30-7.15(\mathrm{~m}$, $3 \mathrm{H}), 4.95-4.83(\mathrm{~m}, 2 \mathrm{H}), 4.32-4.22(\mathrm{~m}, 1 \mathrm{H}), 2.97-2.84(\mathrm{~m}, 1 \mathrm{H}), 2.50-$ 2.32 (m, 2 H), 2.14-2.04 (m, 1 H), 1.85-1.51 (m, 4 H), 1.44-1.17 (m, 1 $\mathrm{H})$. Data (syn) agree with those reported in the literature. ${ }^{11 \mathrm{~b}}$ Detected anti-isomer signal: $\delta=4.69-4.62(\mathrm{~m}, 1 \mathrm{H})$.

${ }^{13} \mathrm{C}$ NMR (101 MHz, $\left.\mathrm{CDCl}_{3}\right)$ (syn): $\delta=211.8,135.5,134.6,130.5,129.0$, $127.5,77.3,51.8,42.9,33.2,28.6,25.4$.

HRMS (ESI): $m / z[M+N a]+$ calcd (as 3:1) for $\mathrm{C}_{14} \mathrm{H}_{16}{ }^{35} \mathrm{ClNNaO}_{3}$ : 304.0711 and $\mathrm{C}_{14} \mathrm{H}_{16}{ }^{37} \mathrm{ClNNaO}_{3}$ : 306.0681; found (as 3:1): $\mathrm{C}_{14} \mathrm{H}_{16}{ }^{35} \mathrm{ClNNaO}_{3}$ : 304.0709 and $\mathrm{C}_{14} \mathrm{H}_{16}{ }^{37} \mathrm{ClNNaO}_{3}$ : 306.0685.

\section{(2R)-2-[(1S)-2-Nitro-1-pyridin-3-ylethyl]cyclohexanone (4l)}

Flash column chromatography (silica gel, gradient hexane-EtOAc); isolated yield: $94 \%$ (58.3 mg from $37.5 \mathrm{mg}$ of alkene); yellow solid; syn/anti: 5.1:1.0; ee (syn): $36 \%$ (by chiral HPLC).

HPLC: CHIRALPAK IA [i-PrOH-hexane (20:80), flow rate: 0.75 $\left.\mathrm{mL} / \mathrm{min}, \lambda=254 \mathrm{~nm}, 25^{\circ} \mathrm{C}\right]$.

IR (ATR): 2941.92, 2863.77, 1705.77, 1549.90, 1428.42, 1379.16, $1130.88,716.55 \mathrm{~cm}^{-1}$.

${ }^{1} \mathrm{H} \mathrm{NMR}\left(400 \mathrm{MHz}, \mathrm{CDCl}_{3}\right.$ ) (syn): $\delta=8.52(\mathrm{dd}, J=4.8,1.7 \mathrm{~Hz}, 1 \mathrm{H}), 8.46$ $(\mathrm{d}, J=2.3 \mathrm{~Hz}, 1 \mathrm{H}), 7.53(\mathrm{dt}, J=7.8,2.0 \mathrm{~Hz}, 1 \mathrm{H}), 7.29-7.25(\mathrm{~m}, 1 \mathrm{H})$, 4.94 (dd, $J=12.9,4.6 \mathrm{~Hz}, 1 \mathrm{H}), 4.68$ (dd, $J=12.9,9.9 \mathrm{~Hz}, 1 \mathrm{H}), 3.80$ (td, $J=9.6,4.6 \mathrm{~Hz}, 1 \mathrm{H}), 2.76-2.65(\mathrm{~m}, 1 \mathrm{H}), 2.53-2.32(\mathrm{~m}, 2 \mathrm{H}), 2.14-2.05$ (m, $1 \mathrm{H}), 1.85-1.50$ (m, $4 \mathrm{H}), 1.25$ (qd, $J=12.7,3.5 \mathrm{~Hz}, 1 \mathrm{H})$. Data (syn) agree with those reported in the literature. ${ }^{11 \mathrm{~d}}$ Detected anti-isomer signals: $\delta=8.54-8.48(\mathrm{~m}, 2 \mathrm{H}), 7.67(\mathrm{dt}, J=7.9,1.9 \mathrm{~Hz}, 1 \mathrm{H}), 4.91-4.83$ (m, $2 \mathrm{H}), 3.94-3.88(\mathrm{~m}, 1 \mathrm{H}), 2.80-2.72(\mathrm{~m}, 1 \mathrm{H}), 2.35-2.23(\mathrm{~m}, 2 \mathrm{H})$, $1.97-1.87(\mathrm{~m}, 1 \mathrm{H}), 1.43-1.31(\mathrm{~m}, 1 \mathrm{H})$.

${ }^{13} \mathrm{C}$ NMR (101 MHz, $\mathrm{CDCl}_{3}$ ) (syn): $\delta=211.2,150.0,149.4,135.8,133.6$, $123.8,78.2,52.3,42.8,41.7,33.3,28.4,25.2$. Detected anti-isomer signals: $\delta=149.13,136.23,123.66,53.33,42.48,41.53,30.77,27.39$.

HRMS (ESI): $m / z[M+H]^{+}$calcd for $\mathrm{C}_{13} \mathrm{H}_{17} \mathrm{~N}_{2} \mathrm{O}_{3}$ : 249.1234; found: $\mathrm{C}_{13} \mathrm{H}_{17} \mathrm{~N}_{2} \mathrm{O}_{3}: 249.1233$.

\section{(2R)-2-[(1R)-2-Nitro-1-(2-thienyl)ethyl]cyclohexanone (4m)}

Flash column chromatography (silica gel, gradient hexane- $\mathrm{Et}_{2} \mathrm{O}$ ); isolated yield: $71 \%$ (44.9 mg from $38.8 \mathrm{mg}$ of alkene); yellow solid; syn/anti: 5.2:1.0; ee (syn): 56\% (by chiral HPLC).

HPLC: CHIRALPAK IA [i-PrOH-hexane (10:90), flow rate: $1 \mathrm{~mL} / \mathrm{min}$, $\left.\lambda=254 \mathrm{~nm}, 25^{\circ} \mathrm{C}\right]$.

IR (ATR): 2939.28, 2863.07, 1705.30, 1550.94 1378.52, 1128.88, $705.58 \mathrm{~cm}^{-1}$.

${ }^{1} \mathrm{H} \mathrm{NMR}\left(400 \mathrm{MHz}, \mathrm{CDCl}_{3}\right.$ ) (syn): $\delta=7.20(\mathrm{dd}, J=5.2,1.3 \mathrm{~Hz}, 1 \mathrm{H}), 6.92$ (dd, $J=5.2,3.5 \mathrm{~Hz}, 1 \mathrm{H}), 6.86(\mathrm{dd}, J=3.5,1.3 \mathrm{~Hz}, 1 \mathrm{H}), 4.88$ (dd, $J=12.7$, $4.7 \mathrm{~Hz}, 1 \mathrm{H}), 4.64(\mathrm{dd}, J=12.7,9.4 \mathrm{~Hz}, 1 \mathrm{H}), 4.12(\mathrm{td}, J=9.1,4.8 \mathrm{~Hz}, 1$ H), 2.71-2.61 (m, $1 \mathrm{H}), 2.51-2.24$ (m, $2 \mathrm{H}), 2.18-2.02$ (m, $1 \mathrm{H}), 1.95-$ 1.78 (m, 2 H), 1.73-1.57 (m, 2 H), 1.47-1.21 (m, 1 H). Data (syn) agree with those reported in the literature ${ }^{18}$ Detected anti-isomer signals: $\delta=7.19(\mathrm{~m}, 1 \mathrm{H}), 4.92-4.75(\mathrm{~m}, 2 \mathrm{H}), 4.23-4.17$ (m, $1 \mathrm{H}), 2.79-2.71$ (m, $1 \mathrm{H})$.

${ }^{13} \mathrm{C}$ NMR (101 MHz, $\mathrm{CDCl}_{3}$ ) (syn): $\delta=211.3,140.6,127.0,126.8,125.1$, 79.3, 53.5, 42.7, 39.5, 32.9, 28.4, 25.2. Detected anti-isomer signals: $\delta=126.88,125.35,78.18,53.56,42.38,39.60,30.77,27.28$.

HRMS (ESI): $m / z$ [M + Na] $]^{+}$calcd for $\mathrm{C}_{12} \mathrm{H}_{15} \mathrm{NNaO}_{3} \mathrm{~S}$ : 276.0665; found: $\mathrm{C}_{12} \mathrm{H}_{15} \mathrm{NNaO}_{3} \mathrm{~S}: 276.0669$.

(2R)-2-[(1S)-1-(2-Chlorophenyl)-2-nitroethyl]cyclobutanone (4n) Flash column chromatography (silica gel, gradient hexane- $\mathrm{Et}_{2} \mathrm{O}$ ); isolated yield: $50 \%$ (31.6 mg from $45.9 \mathrm{mg}$ of alkene); yellow oil; syn/anti: 1.6:1.0; ee (syn): $15 \%$ (by chiral HPLC).

HPLC: CHIRALPAK IC [ $i$-PrOH-hexane (10:90), flow rate: $0.7 \mathrm{~mL} / \mathrm{min}$, $\left.\lambda=210 \mathrm{~nm}, 25^{\circ} \mathrm{C}\right]$.

IR (ATR): 2921.86, 1774.29, 1550.01, 1378.25, 1083.26, 1038.99, $756.18 \mathrm{~cm}^{-1}$.

${ }^{1} \mathrm{H} \mathrm{NMR}\left(400 \mathrm{MHz}, \mathrm{CDCl}_{3}\right)($ syn $): \delta=7.47-7.35(\mathrm{~m}, 1 \mathrm{H}), 7.30-7.15(\mathrm{~m}$, $3 \mathrm{H}), 5.04(\mathrm{dd}, J=12.9,4.6 \mathrm{~Hz}, 1 \mathrm{H}), 4.92-4.80(\mathrm{~m}, 1 \mathrm{H}), 4.29-4.19(\mathrm{~m}$, $1 \mathrm{H}), 3.88-3.76$ (m, $1 \mathrm{H}), 3.19-2.92$ ( $\mathrm{m}, 2 \mathrm{H}), 2.13-1.99$ (m, $1 \mathrm{H}), 1.79-$ $1.60(\mathrm{~m}, 1 \mathrm{H})$. Data $(s y n)$ agree with those reported in the literature. ${ }^{11 \mathrm{~b}}$ anti-Isomer signals: $\delta=7.47-7.35(\mathrm{~m}, 1 \mathrm{H}), 7.30-7.15(\mathrm{~m}, 3 \mathrm{H}), 4.92-$ $4.80(\mathrm{~m}, 2 \mathrm{H}), 4.40$ (q, $J=7.4 \mathrm{~Hz}, 1 \mathrm{H}), 3.88-3.76(\mathrm{~m}, 1 \mathrm{H}), 3.19-2.92$ $(\mathrm{m}, 1 \mathrm{H}), 2.76-2.63(\mathrm{~m}, 1 \mathrm{H}), 2.27-2.16(\mathrm{~m}, 1 \mathrm{H}), 1.79-1.60(\mathrm{~m}, 1 \mathrm{H})$.

${ }^{13} \mathrm{C}$ NMR (101 MHz, $\mathrm{CDCl}_{3}$ ) (syn): $\delta=208.4,134.7,134.2,130.5,129.4$ \& 129.3 syn/anti $_{1}, 128.4,127.7$ \& 127.6 $6_{\text {syn/anti }}, 76.6,60.6,45.2,44.5,16.03$. Detected anti-isomer signals: $\delta=208.17,134.58,130.38,129.35 \&$ $129.32_{\text {syn/anti, }}, 127.66$ \& $127.60_{\text {syn/anti }}, 60.19,14.72$.

HRMS (ESI): $m / z[\mathrm{M}+\mathrm{Na}]^{+}$calcd (as $3: 1$ ) for $\mathrm{C}_{12} \mathrm{H}_{12}{ }^{35} \mathrm{ClNNaO}_{3}$ : 276.0398 and $\mathrm{C}_{12} \mathrm{H}_{12}{ }^{37} \mathrm{ClNNaO}_{3}$ : 278.0368; found (as 3:1): $\mathrm{C}_{12} \mathrm{H}_{12}{ }^{35} \mathrm{ClNNaO}_{3}$ : 276.0401 and $\mathrm{C}_{12} \mathrm{H}_{12}{ }^{37} \mathrm{ClNNaO}_{3}: 278.0377$.

\section{(2R,3R)-2-Methyl-4-nitro-3-(2-thienyl)butanal (40)}

Flash column chromatography (silica gel, gradient hexane- $\mathrm{Et}_{2} \mathrm{O}$ ); isolated yield: $65 \%$ (34.6 mg from $38.8 \mathrm{mg}$ of alkene); yellow oil; syn/anti: 3.7:1.0; ee (syn): $81.8 \%$ (by chiral HPLC).

HPLC: CHIRALPAK IC [ $i$-PrOH-hexane (10:90), flow rate: $1.3 \mathrm{~mL} / \mathrm{min}$, $\left.\lambda=210 \mathrm{~nm}, 25^{\circ} \mathrm{C}\right]$.

IR (ATR): 2973.65, 2730.8, 1722.75, 1553.38, 1379.57, $706.16 \mathrm{~cm}^{-1}$.

${ }^{1} \mathrm{H} \mathrm{NMR}\left(400 \mathrm{MHz}, \mathrm{CDCl}_{3}\right)($ syn) $: \delta=9.68(\mathrm{~s}, 1 \mathrm{H}), 7.25-7.20(\mathrm{~m}, 1 \mathrm{H})$, 6.98-6.85 (m, $2 \mathrm{H}$ ), 4.81-4.59 (m, $2 \mathrm{H}$ ), 4.27-4.19 (m, $1 \mathrm{H}$ ), 2.87-2.72 $(\mathrm{m}, 1 \mathrm{H}), 1.11(\mathrm{~d}, J=7.4 \mathrm{~Hz}, 3 \mathrm{H})$. Data $($ syn) agree with those reported in the literature. ${ }^{19}$ anti-Isomer signals: $\delta=9.60(\mathrm{~s}, 1 \mathrm{H}), 7.25-7.20(\mathrm{~m}$, $1 \mathrm{H}), 6.98-6.85$ (m, $2 \mathrm{H}), 4.81-4.59$ (m, $2 \mathrm{H}$ ), 4.19-4.12 (m, $1 \mathrm{H}), 2.87-$ $2.72(\mathrm{~m}, 1 \mathrm{H}), 1.25(\mathrm{~d}, J=7.4 \mathrm{~Hz}, 3 \mathrm{H})$.

${ }^{13} \mathrm{C}$ NMR $\left(101 \mathrm{MHz}, \mathrm{CDCl}_{3}\right)($ syn $): \delta=201.8,138.9,127.2,126.9,125.4$, 78.5, 48.9, 39.5, 11.6. anti-isomer signals: $\delta=202.14,139.25,127.28$, $126.90,125.53,78.15,49.05,40.17,11.88$.

HRMS (ESI): $m / z$ [M $+\mathrm{Na}]^{+}$calcd for $\mathrm{C}_{9} \mathrm{H}_{11} \mathrm{NNaO}_{3} \mathrm{~S}: 236.0352$; found: $\mathrm{C}_{9} \mathrm{H}_{11} \mathrm{NNaO}_{3} \mathrm{~S}: 236.0362$.

\section{Conflict of Interest}

The authors declare no conflict of interest. 


\section{Funding Information}

We thank The Wild Fund, University of York for funding (B.S.).

\section{Supporting Information}

Supporting information for this article is available online at https://doi.org/10.1055/a-1761-4495.

\section{Primary Data}

Primary data for this article are available online at The York Research Database and can be cited using the following DOI: 10.15124/207e9639-5f06-4eea-af9c-4ded13054afa.

\section{References}

(1) (a) Hajos, Z. G.; Parrish, D. R. J. Org. Chem. 1974, 39, 1615. (b) Eder, U.; Sauer, G.; Wiechert, R. Angew. Chem., Int. Ed. Engl. 1971, 10, 496.

(2) List, B.; Lerner, R. A.; Barbas, C. F. III. J. Am. Chem. Soc. 2000, 122, 2395.

(3) Ahrendt, K. A.; Borths, C. J.; MacMillan, D. W. C. J. Am. Chem. Soc. 2000, $122,4243$.

(4) For examples, see: (a) Mase, N.; Nakai, Y.; Ohara, N.; Yoda, H.; Takabe, K.; Tanaka, F.; Barbas, C. F. III. J. Am. Chem. Soc. 2006, 128, 734. (b) Kinsella, M.; Duggan, P. G.; Lennon, C. M. Tetrahedron: Asymmetry 2011, 22, 1423. (c) Owolabi, I. A.; Subba Reddy, U. V.; Chennapuram, M.; Seki, C.; Okuyama, Y.; Kwon, E.; Uwaai, K.; Tokiwa, M.; Takeshita, M.; Nakano, H. Tetrahedron 2018, 74, 4705.

(5) For examples, see: (a) Steer, A. M.; Bia, N.; Smith, D. K.; Clarke, P. A. Chem. Commun. 2017, 53, 10362. (b) Burroughs, L.; Clarke, P. A.; Forintos, H.; Gilks, J. A. R.; Hayes, C. J.; Vales, M. E.; Wade, W.; Zbytniewski, M. Org. Biomol. Chem. 2012, 10, 1565. (c) Burroughs, L.; Vales, M. E.; Gilks, J. A. R.; Forintos, H.; Hayes, C. J.; Clarke, P. A. Chem. Commun. 2010, 46, 4776.

(6) (a) Cobb, A. J. A.; Shaw, D. M.; Ley, S. V. Synlett 2004, 558. (b) Cobb, A. J. A.; Shaw, D. M.; Longbottom, D. A.; Gold, J. B.; Ley, S. V. Org. Biomol. Chem. 2005, 3, 84.

(7) For several examples see: (a) Franzén, J.; Marigo, M.; Fielenbach, D.; Wabnitz, T. C.; Kjæsgaard, A.; Jørgensen, K. A. J. Am. Chem. Soc. 2005, 127, 18296. (b) Lombardo, M.; Montroni, E.; Quintavalla, A.; Trombini, C. Adv. Synth. Catal. 2012, 354, 3428. (c) McGarraugh, P. G.; Brenner, S. E. Org. Lett. 2009, 11, 5654.
(8) For reviews, see: (a) Valapil, D. G.; Kadagathur, M.; Shankaraiah, N. Eur. J. Org. Chem. 2021, 5288. (b) Yadav, G. D.; Deepa; Singh, S. ChemistrySelect 2019, 4, 5591. (c) Yamashita, Y.; Yasukawa, T.; Yoo, W.-J.; Kitanosono, T.; Kobayashi, S. Chem. Soc. Rev. 2018, 47, 4388.

(9) For a review, see: Merino, P.; Marqués-López, E.; Tejero, T.; Herrera, R. P. Synthesis 2010, 1.

(10) For reviews, see: (a) Albrecht, L.; Krawczyk, H. Wiad. Chem. 2009, 63, 391. (b) Pellissier, H. Curr. Org. Chem. 2018, 22, 323. (c) d'Angelo, J.; Desmaële, D.; Dumas, F.; Guingant, A. Tetrahedron: Asymmetry 1992, 3, 459.

(11) (a) Hayashi, Y.; Gotoh, H.; Hayashi, T.; Shoji, M. Angew. Chem. Int. Ed. 2005, 44, 4212. (b) Pomarański, P.; Czarnocki, Z. Synthesis 2019, 51, 3356. (c) Reyes-Rangel, G.; Vargas-Caporali, J.; Juaristi, E. Tetrahedron 2017, 73, 4707. (d) Mahato, C. K.; Mukherjee, S.; Kundu, M.; Pramanik, A. J. Org. Chem. 2019, 84, 1053. (e) Wiesner, M.; Upert, G.; Angelici, G.; Wennemers, H. J. Am. Chem. Soc. 2010, 132, 6. (f) Wiesner, M.; Revell, J. D.; Wennemers, H. Angew. Chem. Int. Ed. 2008, 47, 1871. (g) Owolabi, I. A.; Chennapuram, M.; Seki, C.; Okuyama, Y.; Kwon, E.; Uwai, K.; Tokiwa, M.; Takeshita, M.; Nakano, H. Bull. Chem. Soc. Jpn. 2019, 92, 696.

(12) List, B.; Pojarliev, P.; Martin, H. J. Org. Lett. 2001, 3, 2423.

(13) (a) Mase, N.; Watanabe, K.; Yoda, H.; Takabe, K.; Tanaka, F.; Barbas, C. F. III. J. Am. Chem. Soc. 2006, 128, 4966. (b) Ishii, T.; Fujioka, S.; Sekiguchi, Y.; Kotsuki, H. J. Am. Chem. Soc. 2004, 126, 9558. (c) Betancort, J. M.; Barbas, C. F. III. Org. Lett. 2001, 3, 3737. (d) Betancort, J. M.; Sakthivel, K.; Thayumanavan, R.; Barbas, C. F. III. Tetrahedron Lett. 2001, 42, 4441.

(14) Vagkidis, N.; Brown, A. J.; Clarke, P. A. Synthesis 2019, 51, 4106.

(15) (a) Mahato, C. K.; Mukherjee, S.; Kundu, M.; Vallapure, V. P.; Pramanik, A. J. Org. Chem. 2021, 86, 5213. (b) Arlegui, A.; Torres, P.; Cuesta, V.; Crusats, J. C.; Moyano, A. Eur. J. Org. Chem. 2020, 4399.

(16) (a) Rani, R.; Peddinti, R. K. Tetrahedron: Asymmetry 2010, 21, 2487. (b) Zhong, J.; Guan, Z.; He, Y.-H. Catal. Commun. 2013, 32, 18.

(17) Kang, I.-J.; Hsu, S.-J.; Yang, H.-Y.; Yeh, T.-K.; Lee, C. C.; Lee, Y.-C.; Tian, Y.-W.; Song, J.-S.; Hsu, T.-A.; Chao, Y.-S.; Yueh, A.; Chern, J.H. J. Med. Chem. 2017, 60, 228-247.

(18) Da Silva, T. L.; Rambo, R. S.; Jacoby, C. G.; Schneider, P. H. Tetrahedron 2020, 76, 130874.

(19) Mossé, S.; Laars, M.; Kriis, K.; Kanger, T.; Alexakis, A. Org. Lett. 2006, 8, 2559. 